\title{
Antagonistic effect of Lactobacillus strains against gas-producing coliforms isolated from colicky infants
}

Francesco Savino ${ }^{1 *}$, Lisa Cordisco ${ }^{2}$, Valentina Tarasco ${ }^{1}$, Emanuela Locatelli ${ }^{1}$, Diana Di Gioia ${ }^{3}$, Roberto Oggero ${ }^{1}$ and Diego Matteuzzi ${ }^{2}$

\begin{abstract}
Background: Infantile colic is a common disturb within the first 3 months of life, nevertheless the pathogenesis is incompletely understood and treatment remains an open issue. Intestinal gas production is thought to be one of the causes of abdominal discomfort in infants suffering from colic. However, data about the role of the amount of gas produced by infants' colonic microbiota and the correlation with the onset of colic symptoms are scanty. The benefit of supplementation with lactobacilli been recently reported but the mechanisms by which they exert their effects have not yet been fully defined. This study was performed to evaluate the interaction between Lactobacillus spp. strains and gas-forming coliforms isolated from stools of colicky infants.

Results: Strains of coliforms were isolated from stools of 45 colicky and 42 control breastfed infants in McConkey Agar and identified using PCR with species-specific primers, and the BBL ${ }^{\mathrm{TM}}$ Enterotube ${ }^{\mathrm{TM}} \|$ system for Enterobacteriaceae. Gas-forming capability of coliforms was assessed in liquid cultures containing lactose as sole carbon source. The average count of total coliforms in colicky infants was significantly higher than controls: 5.98 (2.00-8.76) $\log _{10}$ vs 3.90 (2.50-7.10) CFU/g of faeces ( $p=0.015)$. The following strains were identified: Escherichia coli, Klebsiella pneumoniae, Klebsiella oxytoca, Enterobacter aerogenes, Enterobacter cloacae and Enterococcus faecalis. Then, 27 Lactobacillus strains were tested for their antagonistic effect against coliforms both by halo-forming method and in liquid co-cultures. Lactobacillus delbrueckii subsp.delbrueckii DSM 20074 and L. plantarum MB 456 were able to inhibit all coliforms strains (halo-forming method), also in liquid co-cultures, thus demonstrating an antagonistic activity.

Conclusions: This study shows that two out of 27 strains of Lactobacillus examined possess an antimicrobial effect against six species of gas-forming coliforms isolated from colicky infants. Our findings may stimulate new researches to identify which Lactobacillus strains can improve colicky symptoms by acting on coliforms gut colonization.
\end{abstract}

\section{Background}

The intestinal microbiota exerts many physiological functions such as metabolic and trophic activities and plays an important role in the "barrier effect" against exogenous microbes [1]. It is also involved in the development and activation of the intestinal immune system: a recent study suggests that a more diverse gut microbiota early in life might prevent allergy development [2]. Gut microbiota is acquired during early life and

\footnotetext{
* Correspondence: francesco.savino@unito.it

'Department of Pediatrics, Regina Margherita Children Hospital, P.zza Polonia 94, Torino, 10126, Italy

Full list of author information is available at the end of the article
}

intestinal colonization starts immediately after birth. The ability of species to establish themselves durably in the colonic ecosystem depends on complex interactions between host and bacteria as well as between the bacteria themselves [3]. A wide range of factors may influence the establishment of the intestinal microbiota, including type of delivery, feeding pattern, antibiotic therapy, contact with parents, siblings and hospital staff [4]. The nature of the gut flora, colonic bacterial metabolic pathways, the partial pressure of hydrogen in the colon, the buffering capacity of the colon, and incomplete monosaccharide absorption may play a part in infantile colic. Miller reported an increased breath

C Biomed Central

(c) 2011 Savino et al; licensee BioMed Central Ltd. This is an Open Access article distributed under the terms of the Creative Commons Attribution License (http://creativecommons.org/licenses/by/2.0), which permits unrestricted use, distribution, and reproduction in any medium, provided the original work is properly cited. 
hydrogen excretion in subjects suffering from infantile colic [5]. In 1994, Lehtonen et al. observed that an inadequate lactobacilli level occurring in the first months of life may affect the intestinal fatty acids profile and could favour the development of infantile colic [6]. Treem suggested that colicky infants produce large amounts of gas probably by colonic bacterial fermentation of malabsorbed dietary carbohydrate and that they are relieved of symptoms by the passage of gas [7]. It has also been demonstrated that less methane is produced by stool of colicky infants and this could be due to an inability of the gut microbiota to convert hydrogen to methane with a gastrointestinal hydrogen accumulation [8]. Moreover few old data support the notion that colicky infants produce more breath hydrogen in the fasting state and in response to feedings, which is thought to be evidence of lactose intolerance [9-11].

Differences in gut microbiota have been found among colicky and non-colicky infants: colicky infants are less frequently colonized by Lactobacillus spp. and more frequently by anaerobic gram-negative bacteria [12]. Further, different colonization patterns of lactobacilli have been found among colicky and healthy infants: L. brevis and $L$. lactis are present only in colicky infants while $L$. acidophilus was detected only in healthy ones [13].

The recent finding that $L$. reuteri improve colic symptoms in breastfed infants suggested that a peculiar composition of the intestinal microbiota could favour the development of such disturbance $[14,15]$; however the mechanisms through which lactic acid bacteria act on colic symptoms remain speculative. Moreover, our recent study evaluated the colonization pattern of some important gas-forming coliforms in colicky infants and healthy controls through molecular methods: coliform bacteria, in particular Escherichia coli, resulted more abundant in infants with infantile colic, reinforcing the concept that gut microbiota could be implicated in the aetiopathogenesis of the disturbance $[16,17]$. Nevertheless, up today, little is known about the role of the amount of gas produced by infants' colonic microbiota and the correlation with the onset of colic symptoms, even thought intestinal gas is though to be one of the causes of abdominal discomfort.

This study was performed to elucidate the interaction between lactobacilli and gas-forming coliforms in the gut. To this aim, 27 Lactobacillus strains were examined for their potential in-vitro anti-microbial activity against gas-forming coliforms isolated from stools of colicky infants.

\section{Methods}

\section{Study group and sample collection}

Forty-five breastfed infants suffering from colic symptoms and 42 control breastfed infants (i.e. non colicky) were recruited at the Department of Pediatrics - Regina Margherita Children Hospital, Turin, Italy. They were all aged between 4 and 12 weeks, adequate for gestational age, with a birth weight in the range 2500 and $4000 \mathrm{~g}$, without clinical evidence of chronic illness or gastrointestinal disorders or previous administration of antibiotics and probiotics in the week preceding recruitment. The characteristics of colicky and control subjects are shown in Table 1 . Only exclusively breastfed infants were enrolled in order to reduce variability in the intestinal microflora and in the colonic gas associated with dietary variations $[18,19]$. The colicky cry was defined as a distinctive pain cry difficult to console, lasted for 3 hours or more per day on 3 days or more per week, diagnosed according Wessel criteria [20], with debut $6 \pm$ 1 days before the enrolment. At the enrolment each subject underwent a medical examination and parents were interviewed in order to obtain background data concerning type of delivery, birth weight and gestational age, family history of gastrointestinal disease and atopy. Parents gave written consent to the inclusion of their infants in the study. About 5-10 g faeces were collected from both colicky and non-colicky infants, stored at $80^{\circ} \mathrm{C}$ immediately after collection and subsequently processed. The study was approved by the local ethic committee (Comitato Interaziendale AA.SS.OO. O.I.R.M./S. Anna-Ordine Mauriziano di Torino).

\section{Isolation and identification of coliforms}

Faecal samples, collected from all infants, were homogenized $(10 \%, w / v)$ with sterile saline $(0.9 \% \mathrm{NaCl})$. The homogenates were filtered through a $100 \mu \mathrm{m}$ metal sieve and serially diluted in saline. One hundred $\mu \mathrm{l}$ of each dilution were plated on selective MacConkey Agar (BD Italia, Milan, Italy), which is widely used to isolate enteric bacteria and as a presumptive test for coliform organisms [21] and plates were incubated overnight at $37^{\circ} \mathrm{C}$ in $5 \% \mathrm{CO} 2$ atmosphere. All colonies were counted and counts expressed as $\log 10$ colony-forming units (CFU) per g of faeces. Each isolated strain was subcultured at $37^{\circ} \mathrm{C}$ for 18 $\mathrm{h}$ in Luria Bertani medium (LB) [22] under microaerophilic conditions. Identification of the isolated strains was performed by using the polymerase chain reaction (PCR) technique followed by sequencing of the amplified sequences and the BBL ${ }^{\mathrm{TM}}$ Enterotube ${ }^{\mathrm{TM}}$ II system, which allows the identification of Enterobacteriaceae on the basis of selective carbohydrate fermentation, gas production and the response to selective biochemical reactions (Becton Dickinson GmbH, Heidelberg, Germany). PCR was performed as follows: each isolated strain was streaked on a LB plate, which was incubated overnight at $37^{\circ} \mathrm{C}$. A single colony of each strain was picked and suspended in $20 \mu \mathrm{l}$ of sterile distilled water; the cell suspension was heated at $95^{\circ} \mathrm{C}$ for $10 \mathrm{~min}$ and then cooled to $4^{\circ} \mathrm{C}$. The rDNA 
Table 1 Clinical characteristics of the study population and count of total coliforms bacteria

\begin{tabular}{lccc}
\hline & Colicky infants $(\mathbf{n}=\mathbf{4 5})$ & Controls $(\mathbf{n}=\mathbf{4 2})$ & $\mathbf{p}$-value \\
\hline Gender (M/F) & $25 / 20$ & $24 / 18$ & $1.000^{* *}$ \\
Age at recruitment (days) & $42(15-95)$ & $39(17-98)$ & $0.788^{*}$ \\
Type of delivery (spontaneous/caesarean) & $27 / 18$ & $23 / 19$ & $0.668^{* *}$ \\
Birth weight (grams) & $3300(2550-3970)$ & $3350(2520-4010)$ & $0.951^{*}$ \\
Crying time (minutes per day) & $225(185-310)$ & $105(60-135)$ & $0.000^{*}$ \\
Average count of total coliform bacteria (log10 CFU/g of faeces) & $5.98(2.00-8.76)$ & $3.90(2.50-7.10)$ & $0.015^{*}$ \\
\hline
\end{tabular}

Data are expressed as median (range) or numbers.

*Mann-Whitney Test.

**Fisher's Exact Test

fragment comprising the internal transcribed spacer and the flanking $16 \mathrm{~S}$ and $23 \mathrm{~S}$ rDNA regions was amplified by using the primers indicated in a previous paper [17] and a Biometra (M-Medical SrL, Milan, Italy) thermocycler; the amplified fragments were sequenced and aligned with the most similar ones of GenBank using the Basic Local Alignment Search Tool (BLAST) program.

\section{Evaluation of the gas-forming capability of the isolated strains}

The gas-forming capability of the strains isolated from stool samples was assessed in Lauryl sulphate tryptose broth containing lactose $(10 \mathrm{~g} / \mathrm{L})$ as the sole carbon source. After inoculum and incubation for 24-48 h at $37^{\circ} \mathrm{C}$, bacterial cultures were examined for the presence of gas bubbles in the medium [17]. Production of gas indicated a positive reaction.

\section{Lactobacillus strains and culture conditions}

27 Lactobacillus strains belonging to 8 different species were employed in this work and examined for their anti-microbial activity against coliforms isolated from colicky infants (Table 2). They were obtained from American Type Culture Collection, Manassas, VA, USA (referred to as ATCC strains), German Collection of Microorganisms and Cell Cultures, Braunschweig, Germany (referred to as DSM strains), National Collection of Dairy Organisms, Reading, England (referred to as NCDO strains) and from our collection (Department of Pharmaceutical Sciences, University of Bologna, Italy referred to as $\mathrm{MB}$ or $\mathrm{S}$ strains).

\section{Assessment of the antagonistic activity}

The antagonistic activity of the selected Lactobacillus strains against the isolated coliforms was assayed by using both agar plates and liquid co-cultures of both strains.

\section{- Antimicrobial activity on agar plates}

In this assay both Lactobacillus spp. cells and Lactobacillus neutralized cell-free supernatants (NCS) were employed. Each Lactobacillus strain was grown in MRS broth for $48 \mathrm{~h}$ at $37^{\circ} \mathrm{C}$ in $5 \% \mathrm{CO} 2$ atmosphere and then centrifuged at $15000 \mathrm{~g}$ at $4^{\circ} \mathrm{C}$ for 15 minutes. $\mathrm{pH}$ of the cultures was neutralized to $\mathrm{pH} 7$ with $1 \mathrm{~N} \mathrm{NaOH}$ and cells were separated through filtration (via a $0.2 \mu \mathrm{m}$ pore size filter). Lactobacillus cells were washed twice with saline and suspended in saline at concentrations ranging from $10^{4}$ to $10^{6} \mathrm{CFU} / \mathrm{ml}$. Lactobacillus cells were washed twice with saline and suspended in saline at concentrations of $10^{4}, 10^{5}$ and $10^{6} \mathrm{CFU} / \mathrm{ml}$. All the cell suspensions were assayed to optimize the most suitable cell concentration; the cell concentration of $10^{6}$ $\mathrm{CFU} / \mathrm{ml}$ was then used to perform the comparative assay of the inhibitory activity of the two Lactobacillus strains against coliforms. The paper-disk assay of KirbyBauer [23] was used with some modifications as follows. $50 \mu \mathrm{l}$ of coliform liquid culture in LB broth containing from $10^{3}$ to $10^{6} \mathrm{CFU} / \mathrm{ml}$, in the majority of cases between $10^{5}$ and $10^{6}$, was streaked on a Mac Conkey and LB agar plate; subsequently two sterile paper blank

Table 2 Lactobacillus strains tested for their antagonist activity against coliforms isolated from colicky infants

\begin{tabular}{|c|c|}
\hline $\begin{array}{l}\text { Lactobacillus } \\
\text { species }\end{array}$ & Strains \\
\hline L. acidophilus & $\begin{array}{c}\text { ATCC 11975; MB 252; MB 253; MB 358; MB 359; } \\
\text { MB 422; MB 423; MB 424; MB 425; MB 442; } \\
\text { MB443 }\end{array}$ \\
\hline L. curvatus & MB 67; MB 68 \\
\hline L. casei & ATCC 393; MB 50; MB 441 \\
\hline $\begin{array}{l}\text { L. delbrueckii subsp. } \\
\text { delbrueckii }\end{array}$ & DSM 20074 \\
\hline $\begin{array}{l}\text { L. delbrueckii subsp. } \\
\text { lactis }\end{array}$ & DSM 20076 \\
\hline $\begin{array}{l}\text { L. delbrueckii subsp. } \\
\text { bulgaricus }\end{array}$ & MB 453 \\
\hline $\begin{array}{l}\text { L. salivarius subsp. } \\
\text { salicinius }\end{array}$ & ATCC 11742 \\
\hline $\begin{array}{l}\text { L. salivarius subsp. } \\
\text { salivarius }\end{array}$ & ATCC 11741 \\
\hline L. gasseri & MB 335 \\
\hline L. helveticus & S 36.2; S40.8 \\
\hline L. plantarum & ATCC 8014; NCDO 1193; MB 456 \\
\hline
\end{tabular}


disks (diameter $6 \mathrm{~mm}$ ) were placed on the agar plate and imbibed one with $50 \mu \mathrm{l}$ of washed Lactobacillus cells and the other with $50 \mu$ l of the corresponding NCS. After incubation for $18 \mathrm{~h}$ at $37^{\circ} \mathrm{C}$, the diameters of the inhibition zones were evaluated. The experiments were made in triplicate.

\section{- Antimicrobial activity in liquid co-cultures}

The capability of Lactobacillus DSM 20074 of interfering with the growth of coliforms was evaluated by coincubating both strains. The Lactobacillus strains and the coliform strains were grown on MRS broth and LB broth, respectively. The co-culture experiments was performed in a modified LB medium (i.e. LB additioned with $3 \% \mathrm{w} / \mathrm{v}$ yeast extract) capable of sustaining the growth of both microorganisms. The medium was inoculated with $10^{5} \mathrm{CFU} / \mathrm{ml}$ of both the Lactobacillus and the coliform strains and incubated at $37^{\circ} \mathrm{C}$ in microaerophylic conditions. Controls were prepared by inoculating the same medium either with the Lactobacillus strain or with the coliform one; in addition coliforms were co-cultured with a Lactobacillus strain with no inhibition activity (L. casei MB50, Table 2). At 8-10 h intervals, cultures were centrifuged for $15 \mathrm{~min}$ at $5000 \mathrm{~g}$ and pellets were resuspended in fresh modified LB medium to limit changes in growth due to $\mathrm{pH}$ variation or nutrient limitation. 24 and $48 \mathrm{~h}$ after inoculation, bacterial cells were collected and thoroughly resuspended by vortexing in phosphate-buffered saline (PBS). Thereafter, Lactobacillus and coliform concentrations in the co-cultures and in the controls was determined on MRS agar plates additioned with vancomycin $(0.2 \% \mathrm{w} / \mathrm{v})$ and MacConkey agar plates, which are selective for Lactobacillus spp. and coliforms, respectively. Antimicrobial activity was calculated by comparing the coliform growth in the co-culture and control [8]. Results were expressed as $\log _{10} \mathrm{CFU} / \mathrm{ml}$. The experiment was performed in triplicate.

\section{Statistical Analyses}

Sample size was calculated based on a difference between groups of $1.5 \log 10 \mathrm{CFU} / \mathrm{g}$ faeces. Using $\alpha=$ $0.05, \beta=0.20$ and an estimated standard deviation within groups of $2 \log 10 \mathrm{CFU} / \mathrm{g}$ faeces, 30 patients were needed in each group. Counts $(\log 10 \mathrm{CFU} / \mathrm{g})$ of the total amount of coliform bacteria were calculated for each stool sample. Data are summarized by counts and median and range for categorical and continuous variables respectively. Differences between groups were evaluated with Mann-Whitney's U-test for continuous variables, whereas associations between categorical variables were evaluated with Fisher's exact test. Differences between colicky infants and controls in total amount of each species detected were evaluated with Mann-Whitney's test with Bonferroni correction. Statistical significance was set at a p-value $<0.05$. All statistical calculations were performed with commercially available software (SPSS for Windows release 15Æ0 SPSS Inc., Chicago, IL, USA).

\section{Results}

Isolation and identification of coliforms from colicky infants

Coliform colonies were obtained on MacConkey agar plates from faeces of all the 45 colicky infants and 42 controls. The average count of total coliforms in the 45 faecal samples of colicky infants was 5.98 (2.00-8.76) $\log _{10} \mathrm{CFU} / \mathrm{g}$ of faeces, whereas total coliforms in the control group were 3.90 (2.50-7.10) $\log _{10} \mathrm{CFU} / \mathrm{g}$ of faeces. The difference between the two groups was statistically significant $(p=0.015)$. A total of 145 colonies was randomly picked up from the higher dilutions agar plates $\left(10^{-6}-10^{-8}\right)$ and, only from colicky infants after sub-culturing in LB agar, each purified strain was examined for gas production and characterized at species level by DNA sequencing and carbohydrate fermentation profiling. All isolated strains were found to produce gas from lactose according to the method described above and the BBL ${ }^{\mathrm{TM}}$ Enterotube ${ }^{\mathrm{TM}}$ II system. They were ascribed to six different species (Escherichia coli, Klebsiella oxytoca, Klebsiella pneumoniae, Enterococcus faecalis, Enterobacter aerogenes, and Enterobacter cloacae), as described in Table 3 . The percentage of detection of each species in the faecal samples examined was reported in descending order (Table 3). The same taxonomic identification was obtained with the two methods employed.

\section{Antimicrobial activity of lactic acid bacteria against coliforms}

One strain belonging to each species of isolated coliforms was selected in order to assess the antimicrobial activity of the 27 Lactobacillus strains described in Table 2. The coliform strains were referred to as $E$. coli CG 15b, K. pneumoniae CG 23a, K. oxytoca CG Z, E. aerogenes CG W,E. cloacae CG 6a and E. faecalis CG J.

\begin{tabular}{l} 
Table 3 Identification of the strains isolated from faeces \\
of colicky infants at the species level and \% of each \\
species of the total colonies isolated from the faeces \\
examined \\
\hline \begin{tabular}{lc} 
Coliform identification & Quantitative detection (\%) \\
\hline Escherichia coli & 55.45 \\
Klebsiella oxytoca & 22.15 \\
Klebsiella pneumoniae & 12.34 \\
Enterococcus faecalis & 6.20 \\
Enterobacter aerogenes & 2.70 \\
Enterobacter cloacae & 2.50
\end{tabular}
\end{tabular}


The antagonistic activity was initially examined by using the agar plates method employing both the NCS and washed cells. None of the NCS from all the Lactobacillus strains was found to inhibit the growth of the coliform strains, whereas the washed cells of two strains, i.e. L. delbrueckii subsp.delbrueckii DSM 20074 and L. plantarum MB 456, were found to possess strong inhibitory activity against all 6 coliforms as evidenced by the size of the inhibition halo determined on the coliform plates (Table 4). L. delbrueckii DSM 20074 exhibited a higher anti-bacterial activity against all the coliforms than the MB 456 strain. An example of the halo evidenced on the coliform plates is presented for L. delbrueckii DSM 20074 (Figure 1).

The anti-microbial activity evaluation in liquid cocultures was performed with the Lactobacillus strain showing the highest anti-microbial activity with the previous method, i.e. L. delbrueckii subsp.delbrueckii DSM 20074, and each of the strains referred to the six species of coliform found. Inhibitory activity was evidenced against all the six coliform strains, being higher with the E. coli CG 15b strain. Referring to the experiment with DSM 20074 and E. coli CG 15b strains, the co-culture at the beginning of the incubation time contained $5.43 \pm 0.54 \log _{10} \mathrm{CFU} / \mathrm{ml}$ of $L$. delbrueckii DSM 20074 and $5.70 \pm 0.35 \log _{10} \mathrm{CFU} / \mathrm{ml}$ of E. coli CG $15 \mathrm{~b}$. After $24 \mathrm{~h}$ of incubation, the DSM 20074 concentration was increased to $9.84 \pm 0.94 \log _{10} \mathrm{CFU} / \mathrm{ml}$, whereas no variations were observed in the $E$. coli count. In the parallel control experiment, in which $E$. coli was cultivated with no other strain, the E. coli concentration was $5.65 \pm 0.34$ and $9.00 \pm 1.00 \log _{10}$ $\mathrm{CFU} / \mathrm{ml}$ at the beginning of the incubation and after 24 hours, respectively. When $E$. coli was co-cultured with $L$. casei MB50, no inhibition of E. coli growth was observed. In the co-culture experiments performed with L. delbrueckii DSM20074 and the other coliform strains listed in Table 3, an inhibition of the coliform growth of 3-4 $\log _{10} \mathrm{CFU} / \mathrm{ml}$ was observed (data not shown). On the other hand, the growth of the Lactobacillus strain was never influenced by co-cultivation with the coliform strains.

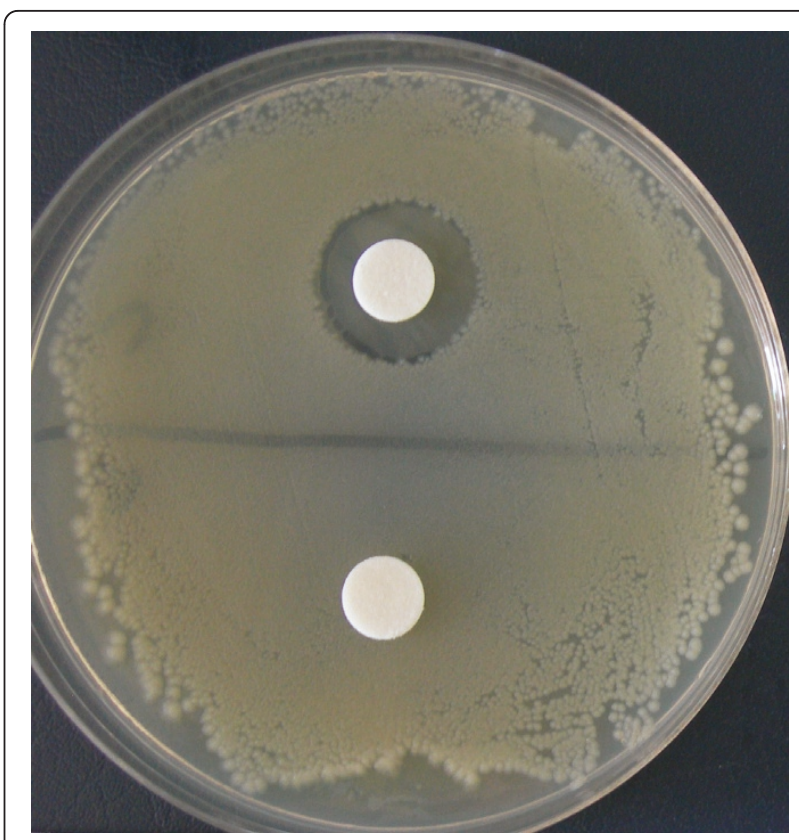

Figure 1 Inhibitory activity of L. delbrueckii DSM 20074 against E. coli CG 15b. Upper paper disk was imbibed with $50 \mu$ of $L$. delbrueckii washed cells, whereas bottom paper disk was imbibed with $50 \mu$ of neutralized supernatant of the same strain

\section{Discussion}

Different studies suggested that colonic gas production favours infantile colic, however the speculation is not supported by well-built scientific researches. Recently, it has been evidenced that gas forming coliform concentration is higher in colicky infants than in healthy controls [16]. Various medical interventions have already been applied to improve symptoms related to infantile colic. Simethicone, a defoaming agent, has been promoted as an effective treatment reducing the formation of intraluminal gas, even though existing data do not demonstrate conclusive benefit of such therapy [24,25]. Alternative solutions to the problem are therefore looked forward.

Recently the benefit of supplementation with Lactobacillus reuteri (American Type Culture Collection Strain

Table 4 Antagonistic activity of L. delbrueckii DSM 20074 and L. plantarum MB 456 cell suspensions (10 ${ }^{6}$ CFU/ml) against coliforms isolated from colicky infants

\begin{tabular}{lcc}
\hline Coliform strains & $\begin{array}{c}\text { Average diameter of the inhibition halo in mm (average } \pm \text { SD) } \\
\text { L. plantarum MB } \mathbf{4 5 6}\end{array}$ \\
\hline E. coli CG 15b & $10.23 \pm 1.29$ & $8.33 \pm 0.89$ \\
K. oxytoca GC Y & $9.75 \pm 1.06$ & $7.75 \pm 0.76$ \\
K. pneumoniae CG 23a & $9.83 \pm 1.04$ & $9.83 \pm 0.64$ \\
E. faecalis GC W & $10.16 \pm 0.76$ & $8.16 \pm 0.56$ \\
E. aerogenes GC K & $10.25 \pm 0.65$ & $7.25 \pm 0.25$ \\
E. cloacae CG 6a & $10.25 \pm 0.35$ & $7.05 \pm 0.35$ \\
\hline
\end{tabular}

It has been expressed as average diameter of inhibition halos obtained on LB agar plates inoculated with each of the selected coliform strains 
55730 and DSM 17 938) has been reported opening a new therapeutic approach $[14,15]$, even though clinical trials are needed to promote new treatments to reduce abdominal pain related to infantile colic [16].

Coliform growth and carbohydrate fermentation affect ammonia absorption and urea nitrogen recycling and excretion. We observed reduction in fecal ammonia concentrations in breastfed infants given L. reuteri and this could be related to modification of bacterial enzyme activity depending on gut microbiota and suggested that gas forming coliforms may be involved in determining colonic fermentation and consequently excessive intraintestinal air load, aerophagia and pain, characteristic symptoms of colic crying, but many aspects of these relationships are still unclear [15]. In the present study we confirmed the higher count of coliforms in colicky infants with respect to non colicky newborns, as already observed in a previous work [17].

Previous studies had shown that some Lactobacillus spp. strains possessed inhibitory activity against E. coli, preventing the binding of enteropathogenic E. coli and other pathogens to intestinal cells [26]. More recently it has been shown that a synbiotic diet containing both prebiotics and probiotics reduces population of intestinal E. coli and the pathogen population in rats [27].

Given these findings, in this work new Lactobacillus strains possessing anti-microbial activity against gas-producing coliforms were searched and the interaction between selected lactobacilli and coliforms was studied.

Coliforms were isolated from stools of colicky infants and characterized taxonomically and for gas production. They were all gas-producing strains and were attributed to 6 different species. The taxonomic identification of the isolated strains and their relative percentage within the coliform group confirmed the results obtained in a previous study, being $E$. coli the most represented species [17]. Two of the 27 lactic acid bacteria assayed in this study, L. delbrueckii subsp.delbrueckii DSM 20074 and $L$. plantarum MB 456, were able to inhibit the growth of gas-forming coliforms belonging to the different species isolated from colicky infants. The extent of the inhibitory activity was similar for all the coliforms assayed (Table 4), although it was higher for the DSM 20074 strain with respect to the other one. Moreover, the capability of the DSM 20074 strain of hindering the growth of coliforms was also observed in a liquid co-culturing assay. Therefore, this strain appears to be a good candidate to relieve symptoms caused by gas-producing coliforms in colicky infants.

The antagonistic activity of the two Lactobacillus strains was only evidenced when harvested cells were applied, whereas the neutralized culture supernatants did not exert any activity on the same coliforms (Figure 1 ). The inhibitory activity of lactic acid bacteria has generally been ascribed to two mechanisms, which can often coexist: i) the production of bacteriocins or bacteriocin-like molecules, which are very often secreted outside the cell $[28,29]$ and ii) the production of inhibitory non proteinaceous metabolites such as organic acids, carbon dioxide, ethanol, hydrogen peroxide and diacetyl, whose anti-microbial action is well known [30]. In addition, Alakomi et al. reported that lactic acid can permeabilize the membrane of Gram negative bacteria by a mechanism of outer membrane disruption [31]. In the case of the two lactic acid bacteria showing inhibitory activity against coliforms in this work, this activity is linked to the presence of the whole cells, although it is not possible to exclude that putative inhibitory molecules are present in the supernatants at such a low concentration that their activity cannot be detected by the assay employed. Therefore, it is not possible to clearly ascribe the inhibitory activity to a defined group of molecules and further studies are necessary to characterize the exact mechanism of inhibition.

\section{Conclusions}

In conclusion, this study confirmed the presence of a greater amount of coliforms in colicky infants with respect to the controls, mainly belonging to the $E$. coli species.

L. delbrueckii subsp.delbrueckii DSM 20074 strain expressed potential for the inhibition of gas-producing coliforms, and thus can be considered a promising candidate for the treatment of colicky symptoms in infants. At present, few data are available on the role of probiotics in colic and the mechanisms by which probiotic bacterial strains antagonise pathogenic gastrointestinal microorganisms or exert other beneficial effects in vivo have not yet been fully defined. Even so, there is a growing interest within clinical medicine in the understanding of the mechanisms through which lactic acid bacteria exert their antagonistic activity against pathogens in the gut. Finally, clinical investigations about invivo efficacy are necessary to confirm the role of Lactobacillus strains as efficacious probiotic treatment to modulate the colonic microbiota in newborns and improve abdominal discomfort due to infantile colic.

\section{Acknowledgements}

We are grateful to Roberto Calabrese for his help in statistical analysis.

\section{Author details}

'Department of Pediatrics, Regina Margherita Children Hospital, P.zza Polonia

94, Torino, 10126, Italy. ²Department of Pharmaceutical Sciences, University of Bologna, via Belmeloro 6, Bologna, 40126, Italy. ${ }^{3}$ Department of Agroenvironmental Sciences and Technologies - Microbiology Area, University of Bologna, Via Fanin 42, 40127, Bologna, Italy.

\section{Authors' contributions}

FS had primary responsibility for the paper and drafted the manuscript. LC performed the molecular analyses. $\mathrm{VT}$ and $\mathrm{EL}$ were responsible for the 
screening of patients, enrolment and outcome assessment. DDG performed the microbiological analyses. RO had primary responsibility for patients enrolled. DM conceived all the study, participated in its design and coordination and helped to draft the manuscript. All authors read and approved the final manuscript.

\section{Competing interests}

The authors declare that they have no competing interests.

Received: 4 January 2011 Accepted: 30 June 2011

Published: 30 June 2011

\section{References}

1. Guarner F: Enteric flora in health and disease. Digestion 2006, 73:5-12.

2. Sjögren $Y M$, Tomicic $S$, Lundberg A, Böttcher MF, Björkstén B, SverremarkEkström $E$, Jenmalm MC: Influence of early gut microbiota on the maturation of childhood mucosal and systemic immune responses. Clin Exp Allergy 2009, 39:1842-51.

3. Penders J, Thijs C, Vink C, Stelma FF, Snijders B, Kummeling I, van den Brandt PA, Stobberingh EE: Factors influencing the competition of intestinal microbiota in early infancy. Pediatrics 2006, 118:511-21.

4. Adlerberth I, Wold AE: Establishment of the gut microbiota in Western infants. Acta Paediatr 2009, 98:229-38

5. Miller JJ, Mc Veagh P, Fleet GH, Petocs P, Brand JC: Breath hydrogen excretion in infants with colic. Arch Dis Child 1989, 64:725-9.

6. Lehtonen L, Korvenranta H, Eerola E: Intestinal microflora in colicky and non-colicky infants: bacterial cultures and gas-liquid chromatography. $J$ Pediatr Gastroenterol Nutr 1994, 19:310-4.

7. Treem WR: Infant colic: a pediatric gastroenterologist's perspective. Pediatr Clin North Am 1994, 41:1121-38.

8. Belson A, Shetty AK, Yorgin PD, Bujanover Y, Peled Y, Dar MH, Reif S: Colonic Hydrogen elimination and methane production in infants with and without colic syndrome. Dig Dis Sci 2003, 48:1762-76.

9. Moore DJ, Robb TA, Davidson GP: Breath hydrogen response to milk containing lactose in colicky and non-colicky infants. J Pediatr 1988, 113:979-84.

10. Miller JJ, Brand JC, MCVeagh P: Breath hydrogen excretion in infants with colic. Arch Dis Child 1990, 65:248.

11. Kanabar D, Randhawa M, Clayton P: Improvement of symptoms in infant colic following reduction of lactose load with lactase. J Hum Nutr Diet 2001, 14:359-63.

12. Savino F, Cresi F, Pautasso S, Palumeri E, Tullio V, Roana J, Silvestro L, Oggero R: Intestinal microflora in breastfed colicky and non-colicky infants. Acta Paediatr 2004, 93:825-9.

13. Savino F, Bailo E, Oggero R, Tullio V, Roana J, Carlone N, Cuffini AM, Silvestro L: Bacterial counts of intestinal Lactobacillus species in infants with colic. Pediatr Allergy Immunol 2005, 16:72-5.

14. Savino F, Pelle E, Palumeri E, Oggero R, Miniero R: Lactobacillus reuteri (American Type Culture Collection Strain 55730) versus simethicone in the treatment of infantile colic: a prospective randomized study. Pediatrics 2007, 119:e124-30.

15. Savino F, Cordisco L, Tarasco V, Palumeri E, Calabrese R, Oggero R, Roos S, Matteuzzi D: Lactobacillus reuteri DSM 17938 in infantile colic: a randomized, double-blind, placebo-controlled trial. Pediatrics 2010, 126: e526-33.

16. Savino F, Tarasco V: New treatments for infantile colic. Curr Opin Pediatr 2010, 22:791-797.

17. Savino F, Cordisco L, Tarasco V, Calabrese R, Palumeri E, Matteuzzi D: Molecular identification of coliform bacteria from colicky breastfed infants. Acta Paediatr 2009, 98:1582-8

18. Jiang T, Suarez FL, Levitt MD, Nelson SE, Ziegler EE: Gas production by feces of infants. J Pediatr Gastroenterol Nutr 2001, 32:534-41.

19. Penders J, Vink C, Driessen C, London N, Thijs C, Stobberingh EE: Quantification of Bifidobacterium spp., Escherichia coli and Clostridium difficile in faecal samples of breast-fed and formula-fed infants by realtime PCR. FEMS Microbiol Lett 2005, 243:141-7.

20. Wessel MA, Cobb JC, Jackson EB, Harris GS, Detwiler AC: Paroxismal fussing in infancy, sometimes called "colic". Pediatrics 1954, 14:421-35

21. Nakamura N, Gaskins HR, Collier CT, Nava GM, Rai D, Petschow B: Molecular ecological analysis of fecal bacterial populations from term infants fed formula supplemented with selected blends of probiotics. App/ Environ Microbiol 2009, 75:1121-8.

22. Sambrook J, Fritsch EF, Maniatis T: Molecular Cloning: A Laboratory Manual. 2 edition. New York: Cold Spring Harbor Laboratory Press; 1989.

23. Bauer AW, Kirby WMM, Sherris JC, Turck M: Antibiotic susceptibility testing by a standardized single disk method. Am J Clin Pathol 1966, 45:493-6.

24. Garrison MM, Christakis DA: A systematic review of treatments for infant colic. Pediatrics 2000, 106:184-90.

25. Lucassen PL, Assendelft WJ, Gubbels JW, van Eijk JT, van Geldrop WJ, Neven AK: Effectiveness of treatments for infantile colic: systematic review. BMJ 1998, 316:1563-9.

26. Servin AL: Antagonistic activities of lactobacilli and bifidobacteria against microbial pathogens. FEMS Microbiol Rev 2004, 28:405-40.

27. Liong MT, Shah NP: Effects of a Lactobacillus casei synbiotic on serum lipoprotein, intestinal microflora, and organic acids in rats. J Dairy SCi 2006, 89:1390-9.

28. Vandenbergh PA: Lactic acid bacteria, their metabolic products and interference with microbial growth. FEMS Microbiol Rev 1993, 12:22-38.

29. Santini C, Baffoni L, Gaggia F, Granata M, Gasbarri R, Di Gioia D, Biavati B: Characterization of probiotic strains: an application as feed additives in poultry against Campylobacter jejuni. Int J Food Microbiol 2010, 141: S98-S108.

30. Galvez A, Abriouel H, Benomar N, Lucas R: Microbial antagonists to foodborne pathogens and biocontrol. Curr Op Biotechnol 2010, 21:142-8.

31. Alakomi HL, Skytta E, Saarela M, Mattila-Sandholm T, Latva-Kala K Helamder IM: Lactic acid permeabilizes Gram-negative bacteria by disrupting the outer membrane. App/ Environ Microbiol 2000, 66:2001-5.

\section{doi:10.1186/1471-2180-11-157}

Cite this article as: Savino et al: Antagonistic effect of Lactobacillus strains against gas-producing coliforms isolated from colicky infants. BMC Microbiology 2011 11:157.

\section{Submit your next manuscript to BioMed Central and take full advantage of:}

- Convenient online submission

- Thorough peer review

- No space constraints or color figure charges

- Immediate publication on acceptance

- Inclusion in PubMed, CAS, Scopus and Google Scholar

- Research which is freely available for redistribution

Submit your manuscript at www.biomedcentral.com/submit
C Biomed Central 